\title{
AN EPIDEMIC MODEL WITH EXPOSURE-DEPENDENT SEVERITIES
}

\author{
FRANK BALL, ${ }^{*}$ The University of Nottingham \\ TOM BRITTON, ${ }^{* *}$ Stockholm University
}

\begin{abstract}
We consider a stochastic model for the spread of a susceptible-infective-removed (SIR) epidemic among a closed, finite population, in which there are two types of severity of infectious individuals, namely mild and severe. The type of severity depends on the amount of infectious exposure an individual receives, in that infectives are always initially mild but may become severe if additionally exposed. Large-population properties of the model are derived. In particular, a coupling argument is used to provide a rigorous branching process approximation to the early stages of an epidemic, and an embedding argument is used to derive a strong law and an associated central limit theorem for the final outcome of an epidemic in the event of a major outbreak. The basic reproduction number, which determines whether or not a major outbreak can occur given few initial infectives, depends only on parameters of the mild infectious state, whereas the final outcome in the event of a major outbreak depends also on parameters of the severe state. Moreover, the limiting final size proportions need not even be continuous in the model parameters.
\end{abstract}

Keywords: Stochastic epidemic; final size; basic reproduction number; infections of varying severity; exposure; branching process; coupling; embedded process; weak convergence; central limit theorem

2000 Mathematics Subject Classification: Primary 92D30

Secondary 60F99; 60K99

\section{Introduction}

This paper is concerned with a susceptible-infective-removed (SIR) epidemic model describing the spread of an infectious disease in a closed, finite community (see, for example, Lefèvre (1990) and Andersson and Britton (2000)). At a meeting in August 2003 in Mariefred, Sweden, attended by epidemiologists, mathematicians, and statisticians, the need for epidemic models allowing different degrees of severity of disease was expressed by several epidemiologists. Examples of diseases for which this is relevant include measles (Aaby et al. (1998), Butler et al. (1994), Morley and Aaby (1997)), varicella (Parang and Archana (2004)), hepatitis-B (Medley et al. (2001)), and dengue fever (Mangada and Igarashi (1998)). Typically, the degree of severity of a case depends on the amount of disease exposure an individual has received, and in severe cases the disease tends to spread more than in mild cases. In the present paper, 'severity' refers to how infectious an individual is and not to the degree of illness, though in reality the two are often correlated. It is the former that is relevant to the spread of the

Received 19 May 2005; revision received 13 July 2005.

* Postal address: School of Mathematical Sciences, The University of Nottingham, University Park, Nottingham NG7 2RD, UK. Email address: frank.ball@ nottingham.ac.uk

** Postal address: Department of Mathematics, Stockholm University, SE-106 91 Stockholm, Sweden.

Email address: tom.britton@math.su.se 
epidemic, which is the focus of the present paper, whereas the latter is of course important from both an individual and a national health perspective.

In this paper, a model which attempts to capture the features of how varying severity affects the spread of a disease in a homogeneously mixing community is defined and studied. The model, which we call the exposure-dependent severity (EDS) model, has two different severities, mild and severe, and is defined without any specific disease in mind. Individuals who become infected are initially mild cases, but they may subsequently become severe cases if they are exposed further to the disease. (The extension to more than two different severities is discussed briefly at the conclusion of the paper.) The large-population behaviour of the EDS model is analysed. The basic reproduction number $R_{0}$, which determines whether a major outbreak is possible given few initial infectives, depends only on parameters governing the mild infectious state. However, the final size in the event of a major outbreak depends also on parameters governing the severe state. Furthermore, the limiting final size as the population becomes infinite depends on the parameters in a surprising way; it is discontinuous in the initial proportion infected as well as in other model parameters.

The paper is organised as follows. The EDS model is defined in Section 2, where both a real-time description and a so-called Sellke construction of the epidemic (Sellke (1983)), which is used to analyse the model, are presented. A heuristic explanation of the final size equations in a large community is given in Section 3, which also contains a numerical example illustrating that the asymptotic final infected proportions of different types may be discontinuous in the model parameters. The asymptotic behaviour of the model as the population size tends to infinity is analysed rigorously in Section 4. In Section 4.1, an embedding argument is used to prove a strong law and an associated central limit theorem for the final outcome of an epidemic initiated by a strictly positive asymptotic proportion of infectives. In Section 4.2, we treat the case of when the number of initial infectives is held fixed as the population size tends to infinity. A coupling argument is used to justify a branching process approximation for the early stages of an epidemic, and the final outcome of an epidemic in the event of a major outbreak is studied using embedding techniques. The paper concludes with a brief discussion in Section 5 .

\section{The EDS epidemic model}

\subsection{Definition of the model}

Consider a closed, homogeneously mixing population consisting initially (i.e. at time $t=0$ ) of $n$ susceptible individuals, $m$ mildly infectious individuals, 0 severely infectious individuals, and 0 removed individuals. A given mildly infectious individual makes contact with any given individual at the points of a homogeneous Poisson process of rate $\lambda_{\mathrm{M}} / n$ throughout an infectious period $I^{(\mathrm{M})}$ having distribution $F_{\mathrm{M}}$. A given severely infectious individual makes contact with any given individual at the points of a homogeneous Poisson process of rate $\lambda_{S} / n$ throughout an infectious period $I^{(\mathrm{S})}$ having distribution $F_{\mathrm{S}}$. Each time a susceptible individual is contacted it becomes mildly infectious with probability $p_{\mathrm{M}}$. Each time a mildly infected individual is contacted it becomes severely infectious with probability $p_{\mathrm{S}}$. Contacts with severely infectious individuals have no effect. All infectious periods, contact processes, and outcomes of contacts are mutually independent. The epidemic continues until there are no mildly or severely infectious individuals in the population.

It is worth emphasising that the mild and severe infectious periods of an individual may or may not overlap, and that, if they overlap, the mild infectious period may end before or after the severe infectious period has ended. To be more specific, if a mildly infectious individual is 
contacted successfully during its mild infectious period, then it contacts any given individual at rate $\left(\lambda_{\mathrm{M}}+\lambda_{\mathrm{S}}\right) / n$ until one of its infectious periods ends. If the mild infectious period ends first, then it contacts any given individual at rate $\lambda_{\mathrm{S}} / n$ until its severe infectious period ends, after which it is removed and plays no further role in the epidemic. If the severe infectious period ends first, then it contacts any given individual at rate $\lambda_{\mathrm{M}} / n$ until its mild infectious period ends, after which it is removed and plays no further role in the epidemic. Alternatively, if the first successful contact with a mildly infectious individual occurs after its mild infectious period has ended, then it still becomes severely infected and again is removed at the end of its severe infectious period.

\subsection{Sellke construction of the model}

We now construct the model above using methods from Sellke (1983). We label the $m$ initial mildly infectious individuals $-(m-1),-(m-2), \ldots, 0$ and the susceptible individuals $1,2, \ldots, n$. For $i=-(m-1),-(m-2), \ldots, n$, let $Q_{i}^{(\mathrm{M})}$ and $Q_{i}^{(\mathrm{S})}$ be random variables exponentially distributed with intensities $p_{\mathrm{M}}$ and $p_{\mathrm{S}}$, respectively, and let $I_{i}^{(\mathrm{M})}$ and $I_{i}^{(\mathrm{S})}$ be random variables distributed according to $F_{\mathrm{M}}$ and $F_{\mathrm{S}}$, respectively. These $4(m+n)$ random variables are mutually independent. The variable $Q_{i}^{(\mathrm{M})}$ is the resistance of individual $i$ against becoming mildly infectious and $Q_{i}^{(\mathrm{S})}$ the extra resistance of individual $i$ against becoming severely infectious once mildly infectious. (Note that $Q_{i}^{(\mathrm{M})}, i=-(m-1),-(m-2), \ldots, 0$, need not be defined, as these individuals are mildly infectious at the start of the epidemic.) The variables $I_{i}^{(\mathrm{M})}$ and $I_{i}^{(\mathrm{S})}$ denote individual $i$ 's mild and severe infectious periods if it becomes so infected.

The epidemic is constructed as follows. At any time $t \geq 0$, individual $i$ accumulates exposure to infection at rate $\left(\lambda_{\mathrm{M}} Y_{\mathrm{M}}(t)+\lambda_{\mathrm{S}} Y_{\mathrm{S}}(t)\right) / n$, where $Y_{\mathrm{M}}(t)$ and $Y_{\mathrm{S}}(t)$ respectively denote the number of mild and severe infectives at time $t$. An initial mild infective, $j$ say, remains so for a period of length $I_{j}^{(\mathrm{M})}$. It becomes severely infectious if and when its accumulated exposure to infection reaches $Q_{j}^{(\mathrm{S})}$ and remains so for a period of length $I_{j}^{(\mathrm{S})}$. Similarly, a susceptible, $i$ say, becomes mildly infectious if and when its accumulated exposure to infection reaches $Q_{i}^{(\mathrm{M})}$ and remains so for a period of length $I_{i}^{(\mathrm{M})}$. If and when individual $i$ 's accumulated exposure to infection reaches $Q_{i}^{(\mathrm{M})}+Q_{i}^{(\mathrm{S})}$, it becomes severely infectious and remains so for a period of length $I_{i}^{(\mathrm{S})}$. (As mentioned above, note that a person can be both mildly and severely infectious at the same time; 'additionally infectious' might thus be a better name for the second state.) The epidemic ceases as soon as there are no mild or severe infectives present in the population, i.e. when $Y_{\mathrm{M}}(t)=Y_{\mathrm{S}}(t)=0$.

Observe that, under the above construction, it is possible for a mild infective to infect itself and hence become severely infected. If this possibility is also allowed in the model described in Section 2.1, then it is easily verified that the Sellke construction yields a process that is probabilistically equivalent to that model. The infectious periods clearly follow the correct distributions and the lack-of-memory property of the exponential distribution ensures that the infection processes are equivalent.

The assumption that an individual can infect itself may appear unrealistic, although it can be thought of as permitting the possibility of a mild infective spontaneously becoming severely infectious. The construction can be modified to exclude this possibility. However, the assumption does not affect the asymptotic behaviour of the model as $n \rightarrow \infty$, and the analysis is simplified by retaining it.

The model can in fact be generalised to allow the contact rate to vary over time without affecting the final size distribution. The random quantities $\lambda_{\mathrm{M}} I^{(\mathrm{M})} / n$ and $\lambda_{\mathrm{S}} I^{(\mathrm{S})} / n$ are the accumulated infection forces exerted on a given individual by a given mild and severe infective, 
respectively. If the contact rate $t$ time units after infection is $\lambda_{\mathrm{M}}(t)$ and $\lambda_{\mathrm{S}}(t)$ for mild and severe infectives, respectively, where the functions $\lambda_{\mathrm{M}}(t)$ and $\lambda_{\mathrm{S}}(t)$ may be deterministic or random, then it is clear from the Sellke construction that the final outcome of this extended epidemic model coincides with that of the previous model, provided that $\lambda_{\mathrm{M}} I^{(\mathrm{M})}$ and $\lambda_{\mathrm{S}} I^{(\mathrm{S})}$ are chosen such that they have the same distributions as $\int_{0}^{\infty} \lambda_{\mathrm{M}}(t) \mathrm{d} t$ and $\int_{0}^{\infty} \lambda_{\mathrm{S}}(t) \mathrm{d} t$, respectively. Thus, for example, a latency period $\left(\lambda_{i}(t)=0\right.$ for $\left.t \leq L_{i}, i=\mathrm{M}, \mathrm{S}\right)$ does not affect the distribution of the final outcome.

\section{Heuristics and a numerical example}

\subsection{Final size equations}

Assume that the initial number of susceptibles $n$ is large, and let $\mu=m / n$. Let $r_{\mathrm{M}}$ denote the proportion of susceptibles who ultimately become mildly infectious and note that some of these may also become severely infectious. Let $r_{\mathrm{S}}$ denote the proportion of susceptibles who ultimately become severely infectious, and let $r_{0}$ denote the proportion of initial mild infectives who become severely infectious during the course of the epidemic. The total force of infection exerted on an individual during the entire epidemic is then given by

$$
\tau=\lambda_{\mathrm{M}} \iota_{\mathrm{M}}\left(\mu+r_{\mathrm{M}}\right)+\lambda_{\mathrm{S}} \iota_{\mathrm{S}}\left(\mu r_{0}+r_{\mathrm{S}}\right),
$$

where $\iota_{\mathrm{M}}$ and $\iota_{\mathrm{S}}$ are the mean infectious periods for mild and severe infectives, respectively. This follows because $n\left(\mu+r_{\mathrm{M}}\right)$ is the total number of mild infectives, each of which has contact with a given other individual at the average accumulated rate $\lambda_{\mathrm{M}} \iota_{\mathrm{M}} / n$; similarly, $n\left(\mu r_{0}+r_{\mathrm{S}}\right)$ is the total number of severe infectives, each of which has contact with a given other individual at the average accumulated rate $\lambda_{\mathrm{S}} \iota_{\mathrm{S}} / n$. A susceptible individual is infected by a single contact with probability $p_{\mathrm{M}}$, so the probability that a given susceptible ultimately becomes mildly infectious is $1-\exp \left[-p_{\mathrm{M}}\left(\lambda_{\mathrm{M}} \iota_{\mathrm{M}}\left(\mu+r_{\mathrm{M}}\right)+\lambda_{\mathrm{S}} \iota_{\mathrm{S}}\left(\mu r_{0}+r_{\mathrm{S}}\right)\right)\right]$, which also gives the proportion of susceptibles who are eventually mildly infected, i.e. $r_{\mathrm{M}}$. To derive an expression for $r_{\mathrm{S}}$, the probability that a susceptible ultimately becomes severely infectious, requires a bit more thought. For this event to happen, a susceptible individual must first become mildly infectious and later severely infectious. From the Sellke construction, this happens if the sum of the mild and severe resistances does not exceed the total force of infection. The mild resistance (denoted by $Q_{i}^{(\mathrm{M})}$ in Section 2.2), i.e. the accumulated force necessary for a susceptible to become mildly infectious, is exponentially distributed with parameter $p_{\mathrm{M}}$. Similarly, the additional severe resistance $Q_{i}^{(\mathrm{S})}$ is exponentially distributed with parameter $p_{\mathrm{S}}$. Thus,

$$
r_{\mathrm{S}}=\mathrm{P}\left[Q_{i}^{(\mathrm{M})}+Q_{i}^{(\mathrm{S})}<\lambda_{\mathrm{M} \iota_{\mathrm{M}}}\left(\mu+r_{\mathrm{M}}\right)+\lambda_{\mathrm{S} \iota_{\mathrm{S}}}\left(\mu r_{0}+r_{\mathrm{S}}\right)\right]
$$

conditioning on $Q_{i}^{(\mathrm{M})}$ yields

$$
r_{\mathrm{S}}= \begin{cases}1-\frac{p_{\mathrm{M}} \mathrm{e}^{-p_{\mathrm{S}} \tau}-p_{\mathrm{S}} \mathrm{e}^{-p_{\mathrm{M}} \tau}}{p_{\mathrm{M}}-p_{\mathrm{S}}} & \text { if } p_{\mathrm{M}} \neq p_{\mathrm{S}}, \\ 1-(1+p \tau) \mathrm{e}^{-p \tau} & \text { if } p_{\mathrm{M}}=p_{\mathrm{S}}=p, \text { say. }\end{cases}
$$

Recall that $\tau$ is defined in (1). Finally, an initial mild infective becomes severely infectious if its severe resistance is less than the total force of infection, which happens with probability $r_{0}=1-\mathrm{e}^{-p_{\mathrm{S}} \tau}$. 
To summarise, we have the following set of balancing equations for the final infected proportions of different types, $r_{\mathrm{M}}, r_{\mathrm{S}}$, and $r_{0}$. If $p_{\mathrm{M}} \neq p_{\mathrm{S}}$ then

$$
\begin{aligned}
& r_{\mathrm{M}}=1-\exp \left[-p_{\mathrm{M}}\left(\lambda_{\mathrm{M}} \iota_{\mathrm{M}}\left(\mu+r_{\mathrm{M}}\right)+\lambda_{\mathrm{S}} \iota_{\mathrm{S}}\left(\mu r_{0}+r_{\mathrm{S}}\right)\right)\right], \\
& r_{\mathrm{S}}=1-\frac{1}{p_{\mathrm{M}}-p_{\mathrm{S}}}\left(p_{\mathrm{M}} \exp \left[-p_{\mathrm{S}}\left(\lambda_{\mathrm{M}} \iota_{\mathrm{M}}\left(\mu+r_{\mathrm{M}}\right)+\lambda_{\mathrm{S}} \iota_{\mathrm{S}}\left(\mu r_{0}+r_{\mathrm{S}}\right)\right)\right]\right. \\
& \left.-p_{\mathrm{S}} \exp \left[-p_{\mathrm{M}}\left(\lambda_{\mathrm{M}} \iota_{\mathrm{M}}\left(\mu+r_{\mathrm{M}}\right)+\lambda_{\mathrm{S}} \iota_{\mathrm{S}}\left(\mu r_{0}+r_{\mathrm{S}}\right)\right)\right]\right), \\
& r_{0}=1-\exp \left[-p_{\mathrm{S}}\left(\lambda_{\mathrm{M}} \iota_{\mathrm{M}}\left(\mu+r_{\mathrm{M}}\right)+\lambda_{\mathrm{S}} \iota_{\mathrm{S}}\left(\mu r_{0}+r_{\mathrm{S}}\right)\right)\right] .
\end{aligned}
$$

If $p_{\mathrm{M}}=p_{\mathrm{S}}=p$ then (3) is replaced by

$$
\begin{aligned}
r_{\mathrm{S}}=1- & \left(1+p\left(\lambda_{\mathrm{M}} \iota_{\mathrm{M}}\left(\mu+r_{\mathrm{M}}\right)+\lambda_{\mathrm{S}} \iota_{\mathrm{S}}\left(\mu r_{0}+r_{\mathrm{S}}\right)\right)\right) \\
& \times \exp \left[-p\left(\lambda_{\mathrm{M}} \iota_{\mathrm{M}}\left(\mu+r_{\mathrm{M}}\right)+\lambda_{\mathrm{S}} \iota_{\mathrm{S}}\left(\mu r_{0}+r_{\mathrm{S}}\right)\right)\right] .
\end{aligned}
$$

Consider a sequence of epidemics, indexed by the initial number of susceptibles $n$, with the $n$th epidemic initially having $m_{n}$ mildly infectious individuals. Let $\mu$ now denote $\lim _{n \rightarrow \infty} n^{-1} m_{n}$, which we assume to exist. Suppose that $\mu>0$. In Corollary 1 , below, we prove that under mild regularity conditions, as $n \rightarrow \infty$, the proportions of the different types ultimately infected converge almost surely to the smallest positive solution of the balancing equations (2) to (4). (As explained in the remark following Corollary 1, if $\left(r_{\mathrm{M}}, r_{\mathrm{S}}, r_{0}\right)$ and $\left(r_{\mathrm{M}}^{\prime}, r_{\mathrm{S}}^{\prime}, r_{0}^{\prime}\right)$ are two positive roots of (2) to (4) satisfying $r_{\mathrm{M}}<r_{\mathrm{M}}^{\prime}$, then $r_{\mathrm{S}}<r_{\mathrm{S}}^{\prime}$ and $r_{0}<r_{0}^{\prime}$. Thus, by the smallest positive root we mean smallest in all components, and such a root is well defined.) We also prove an associated central limit theorem (Theorem 1).

Suppose that $\mu=0$, which would be the case if $m_{n}$ were held fixed as $n \rightarrow \infty$, for example. Note that $r_{0}$ no longer enters the right-hand sides of the equations (2) to (4), although (4) still gives the probability that a given initial mild case ultimately becomes severely infectious. Equations (2) and (3) now admit the solution $r_{\mathrm{M}}=r_{\mathrm{S}}=0$, corresponding to a major outbreak not occurring. If a major outbreak does occur then the asymptotic proportions of different types ultimately infected are given by the smallest strictly positive solution of (2) and (3), and satisfy a central limit theorem (Theorem 3).

Suppose that $m_{n}$ is held fixed as $n \rightarrow \infty$. Then, during the early stages of the epidemic, the probability that a contact is made with a previously infected individual is very small and the process of mild infectives can be approximated by a branching process. Moreover, as $n \rightarrow \infty$, severe cases arise only if the branching process does not become extinct. This approximation is made fully rigorous in Theorem 2 . The offspring distribution of the approximating branching process has mean $R_{0}=\lambda_{\mathrm{M}} \iota_{\mathrm{M}} p_{\mathrm{M}}$, since, as $n \rightarrow \infty$, a given mild infective makes contacts at rate $\lambda_{\mathrm{M}}$ throughout an infectious period having mean $\iota_{\mathrm{M}}$, and each contact is successful with probability $p_{\mathrm{M}}$. Recall that the quantity $R_{0}$ is known as the reproduction number (see, for example, Heesterbeek and Dietz (1996)) and is a threshold parameter for the epidemic, in that if $m$ is small and $n$ large, a major outbreak can occur only if $R_{0}>1$. Note that $R_{0}$ depends only on parameters relating to mild infectives, but that if a major outbreak occurs then its size depends also on parameters relating to severe infectives.

\subsection{A numerical example}

We now illustrate how the deterministic solutions to the balancing equations (2) to (4) may look. We let $\mu=0.01, \lambda_{\mathrm{M}} \iota_{\mathrm{M}}=1, \lambda_{\mathrm{S}} \iota_{\mathrm{S}}=2$, and $p_{\mathrm{M}}=p_{\mathrm{S}}=p$, and examine the solutions $\left(r_{\mathrm{M}}, r_{\mathrm{S}}, r_{0}\right)$ as a function of $p$. In Figure 1 , the equations have been solved numerically and all 


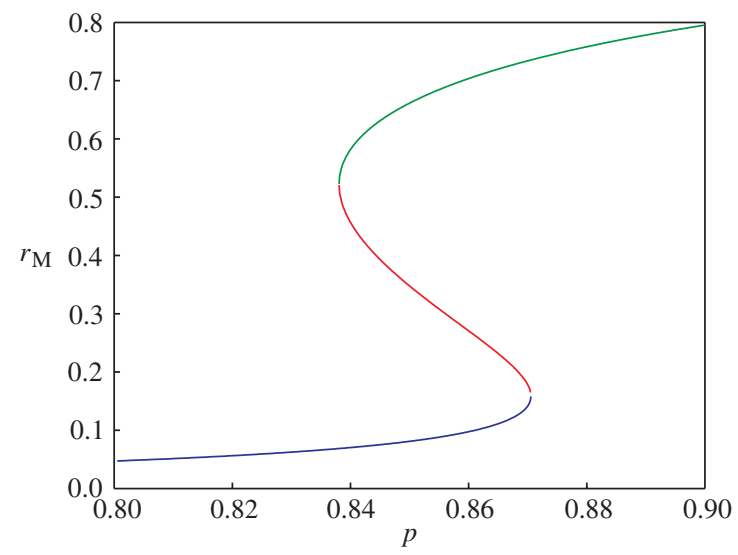

FIGURE 1: All solutions for $r_{\mathrm{M}}$, in the range $0.8 \leq p \leq 0.9$, for the equations (2) to (4), with $\mu=0.01$, $\lambda_{\mathrm{M}} \iota_{\mathrm{M}}=1, \lambda_{\mathrm{S}} \iota_{\mathrm{S}}=2$, and $p_{\mathrm{M}}=p_{\mathrm{S}}=p$.

solutions for $r_{\mathrm{M}}$ are plotted in the range $0.8 \leq p \leq 0.9$ (as might be guessed from the figure, there is only one solution for $p<0.8$ and $p>0.9$ ). As noted above, the final proportion infected converges almost surely to the smallest positive solution to the set of equations (2) to (4). As a consequence, we see that the example exhibits a discontinuity at around $p^{*} \approx 0.8705$. For values of $p$ slightly smaller than this critical value the limiting proportion mildly infected is around 0.158 , whereas for values just above $p^{*}$ the limiting proportion mildly infected suddenly jumps up to around 0.735 ! There are similar discontinuities in $r_{\mathrm{S}}$ and $r_{0}$ (not shown in the figure) for the same value of $p$. There are only discontinuities if both the reproduction number $R_{0}$ is smaller than its threshold of 1 and the epidemic is started with a positive fraction initially infectious (i.e. $\mu>0$ ). The heuristic argument for the discontinuity is that, even though the model is below threshold, the initial proportion mildly infected makes the epidemic 'less subcritical' and, as the parameters move towards criticality (as $p$ grows, in our example), there is a critical point (below threshold!) at which the initial proportion mildly infectious suddenly makes the epidemic supercritical. Similar behaviour was observed by Scalia-Tomba (1985) for an SIR model in which a susceptible has to be successfully contacted twice before it becomes infected, i.e. for an extreme case of the EDS model in which mild infectives make no infectious contacts $\left(\lambda_{\mathrm{M}} \iota_{\mathrm{M}}=0\right)$. Also, in several deterministic models of infectious diseases allowing endemic situations - as opposed to the transient nature of the present SIR model in a closed community - similar discontinuities have been observed, for example by Greenhalgh et al. (2000) and van den Driessche and Watmough (2002). This is known as (backward) bifurcation, referring to the situation in which equations have one solution up until a certain point, at which there suddenly exist more (cf. Figure 1).

We now compare the deterministic solutions discussed above with some simulations of the stochastic model in a finite population. Figure 2 shows the empirical distribution of the final outcome of the stochastic EDS epidemic when $n=1000, m=10, \lambda_{\mathrm{M}}=1, \lambda_{\mathrm{S}}=2$, $p_{\mathrm{M}}=p_{\mathrm{S}}=p$, and $I^{(\mathrm{M})}$ and $I^{(\mathrm{S})}$ are each exponentially distributed with unit mean (i.e. the same parameter values as in the deterministic example). The results are based on 10000 simulations. The upper two figures, showing the numbers of mild infectives, can be compared with the deterministic solutions of Figure 1, evaluated at $p=0.8$ (for the upper-left figure) and $p=0.9$ (for the upper-right figure). Since the epidemic is started with 10 mild infectives it might 

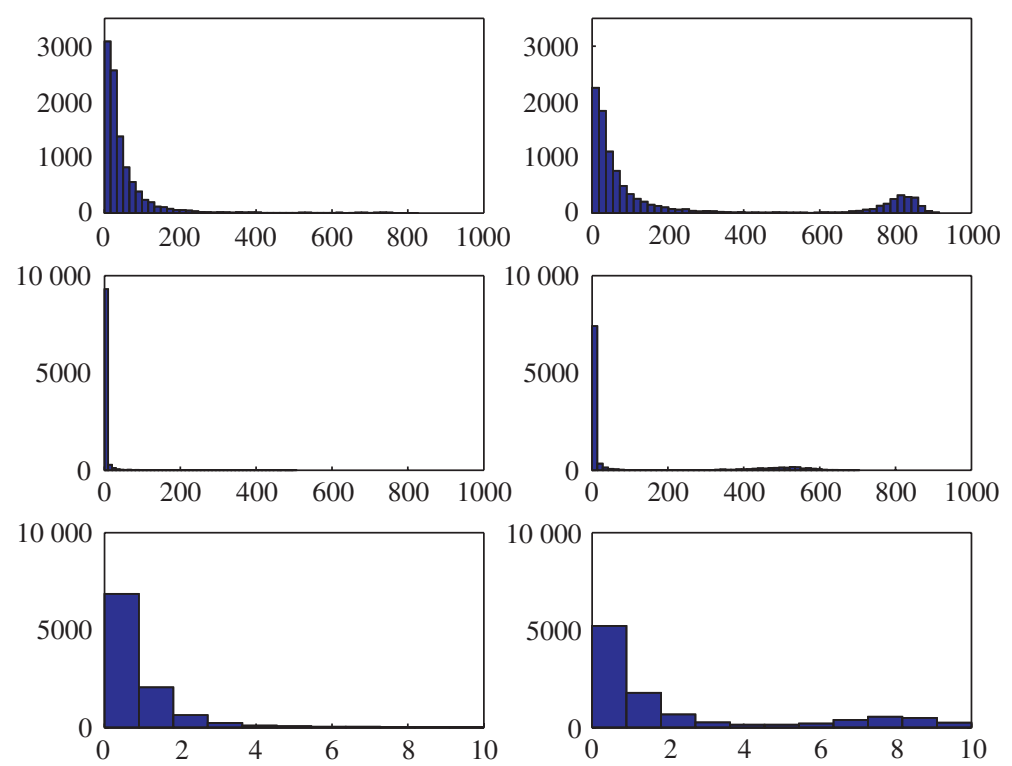

FIGURE 2: The final outcomes of 10000 simulations of the stochastic EDS model for $p=0.8$ (left-hand column) and $p=0.9$ (right-hand column). We plot frequency versus the numbers of mild cases (upper row), severe cases (centre row), and initial cases severely infected (lower row). See the text for further details.

die out quickly for both choices of $p$. This is confirmed by the simulations. In the deterministic solutions of Figure 1 (assuming that an outbreak occurs), it is seen that the ultimate fraction mildly infected is about 0.045 when $p=0.8$ and 0.795 when $p=0.9$. In the simulations for $p=0.8$, nearly all simulations result in small outbreaks, i.e. with an ultimate fraction mildly infected not too far from 0.045 ; however, approximately $1 \%$ of simulations, hardly visible in the figure, have outbreaks in which this fraction is more than 0.5 . On the other hand, in the simulations for $p=0.9$, a greater number of the simulations have large outbreaks with this fraction close to 0.795 , but there are still quite a few small outbreaks. Loosely speaking, the general conclusion in the stochastic setting seems to be that the outbreak size for a given $p$ will be close to the final size solution of the deterministic equation for a possibly slightly different $\tilde{p}$. As a consequence, for values of $p$ close to the bifurcation point, the outbreak may agree with the final size equation for a value $\tilde{p}$ on the other side of the bifurcation point.

\section{Rigorous asymptotic analysis of the model}

In this section, we examine asymptotic properties of the model when $n$ tends to infinity, so we equip all parameters depending on $n$ with an $n$-index. We treat two different initial configurations. Let $\mu_{n}=n^{-1} m_{n}$. Then, either there is a positive proportion of initial mild infectives $\left(\mu_{n} \rightarrow \mu\right.$ as $n \rightarrow \infty$, where $\left.\mu>0\right)$ or else there is a fixed number of initial mild infectives, i.e. $m_{n}=m$ for all $n$. In the first case, we prove a strong law and a central limit theorem for the ultimate proportion of the different types infected. In the second case, we prove a threshold limit theorem indicating that either a minor outbreak occurs, and thus only a few individuals become infected, or else a major epidemic occurs and a more-or-less deterministic fraction of the population ultimately becomes infected. A central limit theorem for the final 
outcome of a major epidemic is also presented. Approximations to the initial stages of the epidemic are obtained by coupling the epidemic process to a suitable branching process and, for the case of a major outbreak, properties of its final outcome are obtained using embedding techniques.

\subsection{The case $\mu_{n} \rightarrow \mu>0$ as $n \rightarrow \infty$}

We study the asymptotic final outcome of the model as $n \rightarrow \infty$ by adapting the embedding argument of Scalia-Tomba (1985), (1990). We use weak convergence in the space of bounded functions on $[0, \infty]$ equipped with the supremum metric. For any real-valued function $f=f(t)$ with domain $[0, \infty]$, let $\|f\|=\sup _{t \in[0, \infty]}|f(t)|$.

Consider the Sellke construction of Section 2.2. For $i=1,2, \ldots, n$ and $t \in[0, \infty]$, let

$$
R_{i}^{(\mathrm{M})}(t)=\mathbf{1}_{\left\{Q_{i}^{(\mathrm{M})} \leq t\right\}}, \quad R_{i}^{(\mathrm{S})}(t)=\mathbf{1}_{\left\{Q_{i}^{(\mathrm{M})}+Q_{i}^{(\mathrm{S})} \leq t\right\}},
$$

and

$$
A_{i}(t)=\lambda_{\mathrm{M}} I_{i}^{(\mathrm{M})} R_{i}^{(\mathrm{M})}(t)+\lambda_{\mathrm{S}} I_{i}^{(\mathrm{S})} R_{i}^{(\mathrm{S})}(t),
$$

where $\mathbf{1}_{\{\cdot\}}$ is an indicator function. Thus, $A_{i}(t)$ is the total force of infection exerted on the population by individual $i$ if it is exposed to $t$ units of infectious pressure. For $i=-\left(m_{n}-1\right)$, $-\left(m_{n}-2\right), \ldots, 0$ and $t \in[0, \infty]$, let

$$
R_{i}^{(0)}(t)=\mathbf{1}_{\left\{Q_{i}^{(\mathrm{S})} \leq t\right\}} \quad \text { and } \quad A_{i}(t)=\lambda_{\mathrm{S}} I_{i}^{(\mathrm{S})} R_{i}^{(0)}(t),
$$

meaning that $A_{i}(t)$ is the severe infectious pressure exerted by initial mild infective $i$ on the population if it is exposed to $t$ units of infectious pressure. For $t \in[0, \infty]$, let

$$
\tilde{A}_{\bullet n}(t)=\sum_{i=-\left(m_{n}-1\right)}^{n} A_{i}(t), \quad R_{\bullet n}^{(\mathrm{M})}(t)=\sum_{i=1}^{n} R_{i}^{(\mathrm{M})}(t), \quad R_{\bullet n}^{(\mathrm{S})}(t)=\sum_{i=1}^{n} R_{i}^{(\mathrm{S})}(t)
$$

and

$$
R_{\bullet n}^{(0)}(t)=\sum_{i=-\left(m_{n}-1\right)}^{0} R_{i}^{(0)}(t) .
$$

The final outcome of the epidemic can be determined as follows. The $m_{n}$ initial mild infectives exert in total $T_{0}^{(n)}=\sum_{i=-\left(m_{n}-1\right)}^{0} \lambda_{\mathrm{M}} I_{i}^{(\mathrm{M})}$ units of infectious pressure on the population from their mild infectious state. These $T_{0}^{(n)}$ units of infectious pressure will create $\tilde{A}_{\bullet n}\left(n^{-1} T_{0}^{(n)}\right)$ further units of infectious pressure, which may in turn create further infectious pressure. For $k=0,1, \ldots$, let

$$
T_{k+1}^{(n)}=T_{0}^{(n)}+\tilde{A}_{\bullet n}\left(n^{-1} T_{k}^{(n)}\right) .
$$

Then $k_{n}^{*}=\min \left\{k: T_{k+1}^{(n)}=T_{k}^{(n)}\right\}$ is well defined since the population is finite. Let $T_{\infty}^{(n)}=T_{k_{n}^{*}}^{(n)}$ and

$$
Z_{n}^{(k)}=R_{\bullet n}^{(k)}\left(n^{-1} T_{\infty}^{(n)}\right), \quad k=0, \mathrm{M}, \mathrm{S}
$$

It is easily verified that $T_{\infty}^{(n)}$ is the total force of infection exerted during the course of the epidemic (also known as the total cost of the epidemic), $Z_{n}^{(0)}$ is the number of initial mild cases that eventually become severe cases, and $Z_{n}^{(\mathrm{M})}$ and $Z_{n}^{(\mathrm{S})}$ are the numbers of susceptible 
individuals that at some time become mild and severe cases, respectively. Note that $Z_{n}^{(\mathrm{M})}$ includes those individuals that eventually go on to become severe cases.

To study the asymptotic behaviour of $\left(Z_{n}^{(0)}, Z_{n}^{(\mathrm{M})}, Z_{n}^{(\mathrm{S})}\right)$, it is convenient to assume that epidemics for different $n$ s are constructed using a common set of random variables

$$
\left\{\left(Q_{i}^{(\mathrm{M})}, Q_{i}^{(\mathrm{S})}, I_{i}^{(\mathrm{M})}, I_{i}^{(\mathrm{S})}\right), i \in \mathbb{Z}\right\}
$$

defined on a probability space $(\Omega, \mathcal{F}, \mathrm{P})$ and distributed as described in Section 2.2. For $t \in[0, \infty]$, let

$$
\begin{aligned}
r_{j}(t) & =\mathrm{E}\left[R_{1}^{(j)}(t)\right], \quad j=\mathrm{M}, \mathrm{S}, \\
r_{0}(t) & =\mu \mathrm{E}\left[R_{0}^{(0)}(t)\right], \\
a(t) & =\mathrm{E}\left[A_{1}(t)\right]=\lambda_{\mathrm{M}} \iota_{\mathrm{M}} r_{\mathrm{M}}(t)+\lambda_{\mathrm{S}} \iota_{\mathrm{S}} r_{\mathrm{S}}(t), \\
a_{0}(t) & =\mu \mathrm{E}\left[A_{0}(t)\right]=\lambda_{\mathrm{S}} \iota_{\mathrm{S}} r_{0}(t),
\end{aligned}
$$

where $\iota_{j}=\mathrm{E}\left[I_{1}^{(j)}\right], j=\mathrm{M}, \mathrm{S}$. It is easily verified that

$$
\begin{aligned}
& r_{0}(t)=\mu\left(1-\mathrm{e}^{-p_{\mathrm{S}} t}\right), \\
& r_{\mathrm{M}}(t)=1-\mathrm{e}^{-p_{\mathrm{M}} t}, \\
& r_{\mathrm{S}}(t)= \begin{cases}1-\frac{p_{\mathrm{M}} \mathrm{e}^{-p_{\mathrm{S}} t}-p_{\mathrm{S}} \mathrm{e}^{-p_{\mathrm{M}} t}}{p_{\mathrm{M}}-p_{\mathrm{S}}} & \text { if } p_{\mathrm{M}} \neq p_{\mathrm{S}}, \\
1-\mathrm{e}^{-p t}(1+p t) & \text { if } p_{\mathrm{M}}=p_{\mathrm{S}}=p .\end{cases}
\end{aligned}
$$

Lemma 1. Suppose that $n^{-1} \mu_{n} \rightarrow \mu$ as $n \rightarrow \infty$, where $\mu>0$, and that $\iota_{j}<\infty, j=\mathrm{M}, \mathrm{S}$. Let $\tilde{a}(t)=a_{0}(t)+a(t)$. Then

$$
\left\|n^{-1} \tilde{A}_{\bullet n}-\tilde{a}\right\| \stackrel{\text { a.s. }}{\longrightarrow} 0 \text { as } n \rightarrow \infty,
$$

where ' $\stackrel{\text { a.s. }}{\longrightarrow}$ denotes almost-sure convergence.

Proof. For $t \in[0, \infty]$,

$$
n^{-1} \tilde{A}_{\bullet n}(t)=\frac{m_{n}}{n} \frac{1}{m_{n}} \sum_{i-\left(m_{n}-1\right)}^{0} A_{i}(t)+\frac{1}{n} \sum_{i=1}^{n} A_{i}(t) \stackrel{\text { a.s. }}{\longrightarrow} \tilde{a}(t) \quad \text { as } n \rightarrow \infty,
$$

by the strong law of large numbers. Thus, there exists an $E \in \mathcal{F}$ such that $\mathrm{P}[E]=1$ and, for all $\omega \in E$,

$$
\lim _{n \rightarrow \infty} n^{-1} \tilde{A}_{\bullet n}(t, \omega)=\tilde{a}(t), \quad t \in(\mathbb{Q} \cap[0, \infty]) \cup\{\infty\},
$$

where $\tilde{A}_{\bullet n}(t, \omega)$ denotes the random variable $\tilde{A}_{\bullet n}(t)$ evaluated at $\omega$. Fix an $\omega \in E$ and an $\varepsilon>0$. Now, $\tilde{a}(0)=0$ and $\tilde{a}(\infty)<\infty$, so, since $\tilde{a}(t)$ is nondecreasing in $t$, there exist a $q \in \mathbb{N}$ and $t_{1}, t_{2}, \ldots, t_{q} \in \mathbb{Q}$ such that $0=t_{0}<t_{1}<t_{2}<\cdots<t_{q}<t_{q+1}=\infty$ and

$$
0<\tilde{a}\left(t_{i}\right)-\tilde{a}\left(t_{i-1}\right)<\frac{1}{2} \varepsilon, \quad i=1,2, \ldots, q+1 .
$$

From (7), there exists an $N \in \mathbb{N}$ such that

$$
\left|n^{-1} \tilde{A}_{\bullet n}\left(t_{i}, \omega\right)-\tilde{a}\left(t_{i}\right)\right|<\frac{1}{2} \varepsilon, \quad i=0,1, \ldots, q+1, n \geq N .
$$


Since $\tilde{A}_{\bullet n}(\cdot, \omega) \equiv \tilde{A}_{\bullet n}(t, \omega)$ is also nondecreasing in $t$, it follows from (8) and (9) that $\left\|n^{-1} \tilde{A}_{\bullet n}(\cdot, \omega)-\tilde{a}(\cdot)\right\|<\varepsilon, n \geq N$. Thus, $\left\|n^{-1} \tilde{A}_{\bullet n}(\cdot, \omega)-\tilde{a}(\cdot)\right\| \rightarrow 0$ as $n \rightarrow \infty$, since $\varepsilon>0$ is arbitrary, and the lemma follows, since $\mathrm{P}[E]=1$.

Corollary 1. Suppose that the conditions of Lemma 1 are satisfied. Let

$$
\tau \equiv \tau(\mu)=\min \left\{t>0: t=\lambda_{\mathrm{M}} \mu \iota_{\mathrm{M}}+\tilde{a}(t)\right\}
$$

and suppose that $\tilde{a}^{\prime}(\tau)<1$, where a prime denotes differentiation. Then $n^{-1} T_{\infty}^{(n)} \stackrel{\text { a.s. }}{\longrightarrow} \tau$ and $n^{-1} Z_{n}^{(i)} \stackrel{\text { a.s. }}{\longrightarrow} r_{i}(\tau), i=0, \mathrm{M}, \mathrm{S}$, as $n \rightarrow \infty$.

Proof. Let $\bar{T}_{k}^{(n)}=n^{-1} T_{k}^{(n)}, k=0,1, \ldots, \infty$. It then follows from (5) and the definition of $T_{\infty}^{(n)}$ that $\bar{T}_{\infty}^{(n)}=\min \left\{t \geq 0: t=\bar{T}_{0}^{(n)}+n^{-1} \tilde{A}_{\bullet n}(t)\right\}$. Since $\tilde{a}^{\prime}$ is continuous on $[0, \infty)$ and $\tilde{a}^{\prime}(\tau)<1$, there exists a $\Delta>0$ such that $t>\lambda_{\mathrm{M}} \mu \iota_{\mathrm{M}}+\tilde{a}(t)$ for all $t \in(\tau, \tau+\Delta)$. Now $\bar{T}_{0}^{(n)} \stackrel{\text { a.s. }}{\longrightarrow} \lambda_{\mathrm{M}} \mu \iota_{\mathrm{M}}$ as $n \rightarrow \infty$, by the strong law of large numbers. In conjunction with Lemma 1, this implies that there exists an $F \in \mathcal{F}$ such that $\mathrm{P}[F]=1$ and, for all $\omega \in F$,

$$
\lim _{n \rightarrow \infty}\left\|n^{-1} \tilde{A}_{\bullet n}(\cdot, \omega)-\tilde{a}(\cdot)\right\|=0 \quad \text { and } \quad \lim _{n \rightarrow \infty} \bar{T}_{0}^{(n)}=\lambda_{\mathrm{M}} \mu \iota_{\mathrm{M}} .
$$

Fix an $\omega \in F$ and an $\varepsilon \in(0, \Delta)$. Let

$$
\begin{aligned}
& C_{1}=\min _{t \in[0, \tau-\varepsilon]}\left\{\lambda_{\mathrm{M}} \mu \iota_{\mathrm{M}}+\tilde{a}(t)-t\right\}, \\
& C_{2}=\tau+\varepsilon-\lambda_{\mathrm{M}} \mu \iota_{\mathrm{M}}-\tilde{a}(\tau+\varepsilon) .
\end{aligned}
$$

Note that the definitions of $\tau$ and $\Delta$ respectively imply that $C_{1}>0$ and $C_{2}>0$. Furthermore, by (10), there exists an $N \in \mathbb{N}$ such that, for all $n \geq N$,

$$
\begin{gathered}
\inf _{t \in[0, \tau-\varepsilon]}\left\{\bar{T}_{0}^{(n)}(\omega)+n^{-1} \tilde{A}_{\bullet n}(t, \omega)-t\right\}>\frac{1}{2} C_{1}, \\
\tau+\varepsilon-\bar{T}_{0}^{(n)}(\omega)-n^{-1} \tilde{A}_{\bullet}(\tau+\varepsilon, \omega)>\frac{1}{2} C_{2},
\end{gathered}
$$

whence $\bar{T}_{\infty}^{(n)}(\omega) \in(\tau-\varepsilon, \tau+\varepsilon)$. Thus, $\bar{T}_{\infty}^{(n)}(\omega) \rightarrow \tau$ as $n \rightarrow \infty$, since $\varepsilon \in(0, \Delta)$ is arbitrary, so

$$
\bar{T}_{\infty}^{(n)} \stackrel{\text { a.s. }}{\longrightarrow} \tau \text { as } n \rightarrow \infty,
$$

since $\mathrm{P}[F]=1$. It is easily seen that results equivalent to (6) hold for $R_{\bullet n}^{(k)}, k=0, \mathrm{M}, \mathrm{S}$, from which it immediately follows that

$$
n^{-1} Z_{n}^{(k)} \stackrel{\text { a.s. }}{\longrightarrow} r_{k}(\tau), \quad k=0, \mathrm{M}, \mathrm{S}, \quad \text { as } n \rightarrow \infty .
$$

Remark 1. It is easily verified that $r_{i}(\tau)=r_{i}, i=\mathrm{M}, \mathrm{S}$, and $r_{0}(\tau)=\mu r_{0}$, where $\left(r_{\mathrm{M}}, r_{\mathrm{S}}, r_{0}\right)$ is the smallest positive solution of the heuristic equations (2) to (4), and that $\tau$ is then given by (1). Note that if $\tau$ and $\tau^{\prime}, \tau<\tau^{\prime}$, are positive roots of $t=\lambda_{\mathrm{M}} \mu \iota_{\mathrm{M}}+\tilde{a}(t)$, then $r_{i}(\tau)<r_{i}\left(\tau^{\prime}\right)$, $i=\mathrm{M}, \mathrm{S}, 0$, meaning that the smallest positive root of (2) to (4) is well defined.

We now derive a central limit theorem for the final outcome of the epidemic. Before stating the main result some more notation is required. For $t \in[0, \infty]$, let $\boldsymbol{X}_{n}(t)=\left(\tilde{A}_{\bullet n}(t), R_{\bullet n}^{(0)}(t)\right.$, 
$\left.R_{\bullet n}^{(\mathrm{M})}(t), R_{\bullet n}^{(\mathrm{S})}(t)\right)^{\top}$ and $\boldsymbol{\Sigma}(t)=\lim _{n \rightarrow \infty} n^{-1} \operatorname{cov}\left(\boldsymbol{X}_{n}(t)\right)$, where, for a vector-valued random variable $\boldsymbol{X}$, say, $\operatorname{cov}(\boldsymbol{X})$ denotes the variance-covariance matrix of $\boldsymbol{X}$. Write $\boldsymbol{\Sigma}(t)$ as

$$
\boldsymbol{\Sigma}(t)=\left[\begin{array}{cccc}
\tilde{\sigma}_{A}^{2}(t) & \sigma_{A 0}(t) & \sigma_{A \mathrm{M}}(t) & \sigma_{A \mathrm{~S}}(t) \\
\sigma_{A 0}(t) & \sigma_{0}^{2}(t) & \sigma_{0 \mathrm{M}}(t) & \sigma_{0 \mathrm{~S}}(t) \\
\sigma_{A \mathrm{M}}(t) & \sigma_{0 \mathrm{M}}(t) & \sigma_{\mathrm{M}}^{2}(t) & \sigma_{\mathrm{MS}}(t) \\
\sigma_{A \mathrm{~S}}(t) & \sigma_{0 \mathrm{~S}}(t) & \sigma_{\mathrm{MS}}(t) & \sigma_{\mathrm{S}}^{2}(t)
\end{array}\right]
$$

Let $\sigma_{i}^{2}=\operatorname{var}\left(I_{1}^{(i)}\right), i=\mathrm{M}, \mathrm{S}$. Elementary calculations yield that

$$
\begin{aligned}
& \sigma_{0}^{2}(t)=r_{0}(t)\left(1-\mu^{-1} r_{0}(t)\right), \quad \sigma_{i}^{2}(t)=r_{i}(t)\left(1-r_{i}(t)\right), \quad i=\mathrm{M}, \mathrm{S}, \\
& \sigma_{0 \mathrm{M}}(t)=\sigma_{0 \mathrm{~S}}(t)=0, \quad \sigma_{\mathrm{MS}}(t)=r_{\mathrm{S}}(t)\left(1-r_{\mathrm{M}}(t)\right), \\
& \sigma_{A 0}(t)=\lambda_{\mathrm{S}} \iota_{\mathrm{S}} \sigma_{0}^{2}(t), \quad \sigma_{A \mathrm{M}}(t)=\lambda_{\mathrm{M}} \iota_{\mathrm{M}} \sigma_{\mathrm{M}}^{2}(t)+\lambda_{\mathrm{S}} \iota_{\mathrm{S}} \sigma_{\mathrm{MS}}(t), \\
& \sigma_{A \mathrm{~S}}(t)=\lambda_{\mathrm{M}} \iota_{\mathrm{M}} \sigma_{\mathrm{MS}}(t)+\lambda_{\mathrm{S}} \iota_{\mathrm{S}} \sigma_{\mathrm{S}}^{2}(t), \\
& \tilde{\sigma}_{A}^{2}(t)=\lambda_{\mathrm{S}}^{2}\left(\sigma_{\mathrm{S}}^{2} r_{0}(t)+\iota_{\mathrm{S}}^{2} \sigma_{0}^{2}(t)\right)+\lambda_{\mathrm{M}}^{2}\left(r_{\mathrm{M}}(t) \sigma_{\mathrm{M}}^{2}+\iota_{\mathrm{M}}^{2} \sigma_{\mathrm{M}}^{2}(t)\right) \\
& +\lambda_{\mathrm{S}}^{2}\left(r_{\mathrm{S}}(t) \sigma_{\mathrm{S}}^{2}+\iota_{\mathrm{S}}^{2} \sigma_{\mathrm{S}}^{2}(t)\right)+2 \lambda_{\mathrm{M}} \lambda_{\mathrm{S}} \iota_{\mathrm{M}} \iota_{\mathrm{S}} \sigma_{\mathrm{MS}}(t) .
\end{aligned}
$$

For $t \in[0, \infty]$, let $\boldsymbol{B}(t)=\left[b_{i j}(t)\right]$ be the $4 \times 4$ matrix with elements given by

$$
\begin{aligned}
& b_{11}(t)=\left(1-\tilde{a}^{\prime}(t)\right)^{-1} \tilde{a}^{\prime}(t), \\
& b_{21}(t)=\left(1-\tilde{a}^{\prime}(t)\right)^{-1} r_{0}^{\prime}(t), \\
& b_{31}(t)=\left(1-\tilde{a}^{\prime}(t)\right)^{-1} r_{\mathrm{M}}^{\prime}(t), \\
& b_{41}(t)=\left(1-\tilde{a}^{\prime}(t)\right)^{-1} r_{\mathrm{S}}^{\prime}(t), \\
& b_{i j}(t)=0, \quad j>1 .
\end{aligned}
$$

Let $\boldsymbol{J}$ be the $4 \times 4$ matrix with elements all equal to 1 , and let $\boldsymbol{I}$ be an identity matrix whose dimension is apparent from its context. For $n=1,2, \ldots$, let $Z_{n}=\left(\tilde{A}_{\bullet}\left(\bar{T}_{\infty}^{(n)}\right), Z_{n}^{(0)}, Z_{n}^{(\mathrm{M})}, Z_{n}^{(\mathrm{S})}\right)^{\top}$, and, for $t \in[0, \infty]$, let $\mu_{Z}(t)=\left(\tilde{a}(t), r_{0}(t), r_{1}(t), r_{2}(t)\right)^{\top}$. By $\stackrel{\text { D }}{\rightarrow}$ ' we denote convergence in distribution.

Theorem 1. Suppose that the conditions of Lemma 1 and Corollary 1 are satisfied, that $\sigma_{i}^{2}<\infty, i=\mathrm{M}, \mathrm{S}$, and that $n^{1 / 2}\left(\mu_{n}-\mu\right) \rightarrow 0$ as $n \rightarrow \infty$. Then $n^{-1 / 2}\left(\boldsymbol{Z}_{n}-n \boldsymbol{\mu}_{Z}(\tau)\right) \stackrel{\mathrm{D}}{\rightarrow} \boldsymbol{Z}$ as $n \rightarrow \infty$, where $\boldsymbol{Z}$ is a four-dimensional zero-mean normal random vector with variancecovariance matrix given by

$$
\boldsymbol{\Sigma}_{Z}=(\boldsymbol{I}+\boldsymbol{B}(\tau)) \boldsymbol{\Sigma}(\tau)\left(\boldsymbol{I}+\boldsymbol{B}(\tau)^{\top}\right)+\mu \lambda_{\mathrm{M}}^{2} \sigma_{\mathrm{M}}^{2} \boldsymbol{B}(\tau) \boldsymbol{J} \boldsymbol{B}(\tau)^{\top} .
$$

Proof. For $t \in[0, \infty]$, let $A_{\bullet n}(t)=\sum_{i=1}^{n} A_{i}(t)$ and $A_{\bullet n}^{(0)}(t)=\sum_{i=-\left(m_{n}-1\right)}^{0} A_{i}(t)$. The sample paths $t \mapsto A_{1}(t)$ are càdlàg (continuous from the right with left limits) and nondecreasing, so, by Example 2.11.16 of van der Vaart and Wellner (1996),

$$
n^{-1 / 2}\left(A_{\bullet n}-\mathrm{E}\left[A_{\bullet}\right]\right) \stackrel{\mathrm{W}}{\rightarrow} X \quad \text { as } n \rightarrow \infty,
$$

where $X$ is a zero-mean Gaussian process and $\stackrel{\text { w }}{\rightarrow}$ ' denotes weak convergence. A result equivalent to (12) holds for each of $A_{\bullet}^{(0)}$ and $R_{\bullet n}^{(k)}, k=0, \mathrm{M}, \mathrm{S}$. For $t \in[0, \infty]$, let $X_{\bullet n}(t)=\left(\tilde{A}_{\bullet n}(t), R_{\bullet n}^{(0)}(t), R_{\bullet n}^{(\mathrm{M})}(t), R_{\bullet n}^{(\mathrm{S})}(t)\right)^{\top}$. Then

$$
n^{-1 / 2}\left(X_{\bullet n}-\mathrm{E}\left[\boldsymbol{X}_{\bullet n}\right]\right) \stackrel{\mathrm{W}}{\rightarrow} \boldsymbol{X} \quad \text { as } n \rightarrow \infty
$$


where $\boldsymbol{X}$ is a four-dimensional zero-mean Gaussian process whose covariance function satisfies $\operatorname{cov}(\boldsymbol{X}(t))=\boldsymbol{\Sigma}(t), t \in[0, \infty]$. (It is easily seen that the finite-dimensional distributions of $n^{-1 / 2}\left(\boldsymbol{X}_{\bullet n}-\mathrm{E}\left[\boldsymbol{X}_{\bullet}\right]\right)$ converge to those of $\boldsymbol{X}$ by using the Cramér-Wold device, and asymptotic tightness of $n^{-1 / 2}\left(\boldsymbol{X}_{\bullet n}-\mathrm{E}\left[\boldsymbol{X}_{\bullet n}\right]\right)$ follows from Lemma 1.4.3 and Theorem 1.5.4 of van der Vaart and Wellner (1996).) Corollary 1 implies that $\bar{T}_{\infty}^{(n)} \stackrel{\mathrm{P}}{\rightarrow} \tau$ as $n \rightarrow \infty$, where ' $\stackrel{\mathrm{P}}{\rightarrow}$ ' denotes convergence in probability. Therefore, by Slutsky's lemma and the continuous mapping theorem (Example 1.4.7 and Theorem 1.3.6 of van der Vaart and Wellner (1996), respectively), it follows from (13) that

$$
n^{-1 / 2}\left(\boldsymbol{X}_{\bullet n}\left(\bar{T}_{\infty}^{(n)}\right)-\mathrm{E}\left[\boldsymbol{X}_{\bullet}\right]\left(\bar{T}_{\infty}^{(n)}\right)\right) \stackrel{\mathrm{D}}{\rightarrow} \boldsymbol{X}(\tau) \quad \text { as } n \rightarrow \infty
$$

Now,

$$
n^{-1 / 2}\left(\tilde{A}_{\bullet n}\left(\bar{T}_{\infty}^{(n)}\right)-n \tilde{a}(\tau)\right)=n^{-1 / 2}\left(\tilde{A}_{\bullet n}\left(\bar{T}_{\infty}^{(n)}\right)-n \tilde{a}\left(\bar{T}_{\infty}^{(n)}\right)\right)+n^{1 / 2}\left(\tilde{a}\left(\bar{T}_{\infty}^{(n)}\right)-\tilde{a}(\tau)\right) .
$$

By the mean value theorem, $n^{1 / 2}\left(\tilde{a}\left(\bar{T}_{\infty}^{(n)}\right)-\tilde{a}(\tau)\right)=n^{1 / 2}\left(\bar{T}_{\infty}^{(n)}-\tau\right) \tilde{a}^{\prime}\left(\eta_{n}\right)$ for some $\eta_{n}$ lying between $\bar{T}_{\infty}^{(n)}$ and $\tau$. Recall that $\bar{T}_{\infty}^{(n)}=\bar{T}_{0}^{(n)}+n^{-1} \tilde{A}_{\bullet n}\left(\bar{T}_{\infty}^{(n)}\right)$ and $\tau=\lambda_{\mathrm{M}} \mu \iota_{\mathrm{M}}+\tilde{a}(\tau)$. Thus,

$$
n^{1 / 2}\left(\bar{T}_{\infty}^{(n)}-\tau\right)=n^{1 / 2}\left(\bar{T}_{0}^{(n)}-\lambda_{\mathrm{M}} \mu \iota_{\mathrm{M}}\right)+n^{-1 / 2}\left(\tilde{A}_{\bullet n}\left(\bar{T}_{\infty}^{(n)}\right)-n \tilde{a}(\tau)\right),
$$

and it follows that

$$
\begin{aligned}
& \left(1-\tilde{a}^{\prime}\left(\eta_{n}\right)\right) n^{-1 / 2}\left(\tilde{A}_{\bullet n}\left(\bar{T}_{\infty}^{(n)}\right)-n \tilde{a}(\tau)\right) \\
& \quad=\tilde{a}^{\prime}\left(\eta_{n}\right) n^{1 / 2}\left(\bar{T}_{0}^{(n)}-\lambda_{\mathrm{M}} \mu \iota_{\mathrm{M}}\right)+n^{-1 / 2}\left(\tilde{A}_{\bullet n}\left(\bar{T}_{\infty}^{(n)}\right)-n \tilde{a}\left(\bar{T}_{\infty}^{(n)}\right)\right) .
\end{aligned}
$$

Now,

$$
n^{-1 / 2}\left(\mathrm{E}\left[\tilde{A}_{\bullet}\right]\left(\bar{T}_{\infty}^{(n)}\right)-n \tilde{a}\left(\bar{T}_{\infty}^{(n)}\right)\right)=n^{1 / 2}\left(n^{-1} m_{n}-\mu\right) \mu^{-1} a_{0}\left(\bar{T}_{\infty}^{(n)}\right) \stackrel{\mathrm{P}}{\rightarrow} 0 \quad \text { as } n \rightarrow \infty,
$$

since $n^{1 / 2}\left(n^{-1} m_{n}-\mu\right) \rightarrow 0$ and $\bar{T}_{\infty}^{(n)} \stackrel{\mathrm{P}}{\rightarrow} \tau$ as $n \rightarrow \infty$. Furthermore, by the central limit theorem,

$$
n^{1 / 2}\left(\bar{T}_{0}^{(n)}-\lambda_{\mathrm{M}} \mu \iota_{\mathrm{M}}\right) \stackrel{\mathrm{D}}{\rightarrow} W_{0} \quad \text { as } n \rightarrow \infty,
$$

where $W_{0}$ is independent of $\boldsymbol{X}(\tau)$ and is normally distributed as follows: $W_{0} \sim \mathcal{N}\left(0, \mu \lambda_{\mathrm{M}}^{2} \sigma_{\mathrm{M}}^{2}\right)$. For $t \in[0, \infty]$, write

$$
\boldsymbol{X}(t)=\left(X_{\tilde{A}}(t), X_{R}^{(0)}(t), X_{R}^{(\mathrm{M})}(t), X_{R}^{(\mathrm{S})}(t)\right)^{\top} .
$$

Now $\eta_{n} \stackrel{\mathrm{P}}{\rightarrow} \tau$ as $n \rightarrow \infty$, so, since $\tilde{a}(t)$ is continuously differentiable and $\tilde{a}^{\prime}(\tau)<1$, it follows from (15) and Slutsky's theorem that

$$
n^{-1 / 2}\left(\tilde{A}_{\bullet}\left(\bar{T}_{\infty}^{(n)}\right)-n \tilde{a}(\tau)\right) \stackrel{\mathrm{D}}{\rightarrow}\left(1-\tilde{a}^{\prime}(\tau)\right)^{-1}\left(\tilde{a}^{\prime}(\tau) W_{0}+X_{A}(\tau)\right) \quad \text { as } n \rightarrow \infty .
$$

Using (14), it then follows that

$$
n^{1 / 2}\left(\bar{T}_{\infty}^{(n)}-\tau\right) \stackrel{\mathrm{D}}{\rightarrow}\left(1-\tilde{a}^{\prime}(\tau)\right)^{-1}\left(W_{0}+X_{A}(\tau)\right) \quad \text { as } n \rightarrow \infty .
$$

For $k=0, \mathrm{M}, \mathrm{S}$, we have

$$
n^{-1 / 2}\left(R_{\bullet n}^{(k)}\left(\bar{T}_{\infty}^{(n)}\right)-n r_{k}(\tau)\right)=n^{-1 / 2}\left(R_{\bullet n}^{(k)}\left(\bar{T}_{\infty}^{(n)}\right)-n r_{k}\left(\bar{T}_{\infty}^{(n)}\right)\right)+n^{1 / 2}\left(r_{k}\left(\bar{T}_{\infty}^{(n)}\right)-r_{k}(\tau)\right)
$$


By applying the mean value theorem to the final term in (18), using (17), and arguing as above, we find that, as $n \rightarrow \infty$,

$$
n^{-1 / 2}\left(R_{\bullet n}^{(k)}\left(\bar{T}_{\infty}^{(n)}\right)-n r_{k}(\tau)\right) \stackrel{\mathrm{D}}{\rightarrow} X_{R}^{(k)}(\tau)+\left(1-a^{\prime}(\tau)\right)^{-1} r_{k}^{\prime}(\tau)\left(W_{0}+X_{A}(\tau)\right) .
$$

Recall that $Z_{n}^{(k)}=R_{\bullet n}^{(k)}\left(\bar{T}_{\infty}^{(n)}\right), k=0, \mathrm{M}, \mathrm{S}$. It follows from (16) and (19) that

$$
n^{-1 / 2}\left(\boldsymbol{Z}_{n}-n \boldsymbol{\mu}_{Z}(\tau)\right) \stackrel{\mathrm{D}}{\rightarrow} \boldsymbol{Z} \quad \text { as } n \rightarrow \infty,
$$

where $\boldsymbol{Z}=(\boldsymbol{I}+\boldsymbol{B}(\tau)) \boldsymbol{X}(\tau)+\boldsymbol{B}(\tau) \boldsymbol{W}$ and $\boldsymbol{W}=\left(W_{0}, W_{0}, W_{0}, W_{0}\right)^{\top}$. Theorem 1 follows by noting that $\boldsymbol{X}(\tau)$ and $\boldsymbol{W}$ are independent.

\subsection{The case $m_{n}=m$ for all $n$}

We study the initial behaviour of the epidemic by adapting the coupling argument of Ball and Donnelly (1995) to the present model. Let $(\Omega, \mathcal{F}, \mathrm{P})$ be a probability space on which are defined the following independent sets of random quantities.

(i) $\mathcal{H}_{i}=\left(I_{i}^{(\mathrm{M})}, I_{i}^{(\mathrm{S})}, \eta_{i}^{(\mathrm{M})}, \eta_{i}^{(\mathrm{S})}, \xi_{i}^{(\mathrm{M})}, \xi_{i}^{(\mathrm{S})}\right), i=-(m-1),-(m-2), \ldots$ These are independent and identically distributed according to $\mathcal{H}=\left(I^{(\mathrm{M})}, I^{(\mathrm{S})}, \eta^{(\mathrm{M})}, \eta^{(\mathrm{S})}, \xi^{(\mathrm{M})}, \xi^{(\mathrm{S})}\right)$, where the components of $\mathcal{H}$ are independent, $I^{(k)}, k=\mathrm{M}, \mathrm{S}$, is distributed according to $F_{k}$, and $\eta^{(\mathrm{M})}, \eta^{(\mathrm{S})}, \xi^{(\mathrm{M})}$, and $\xi^{(\mathrm{S})}$ are homogeneous Poisson processes on $[0, \infty)$ with respective rates $\lambda_{\mathrm{M}}, \lambda_{\mathrm{S}}, \lambda_{\mathrm{M}}$, and $\lambda_{\mathrm{S}}$.

(ii) $\chi_{i}^{(n)}, n=1,2, \ldots, i=1,2, \ldots$ For each $n=1,2, \ldots$, the $\chi_{i}^{(n)}, i=1,2, \ldots$ are independent and uniformly distributed on $\{1,2, \ldots, n\}$.

(iii) $\tilde{\chi}_{i}, i=1,2, \ldots$ These are independent and uniformly distributed on $\{-(m-1)$, $-(m-2), \ldots, 0\}$.

(iv) $U_{i}, i=1,2, \ldots$ These are independent and uniformly distributed on $(0,1)$.

Denote the epidemic described in Section 2.1 by $E_{n}$. A realisation of $E_{n}$ can be constructed as follows. For $i=-(m-1),-(m-2), \ldots, 0$, during its mild infectious period, having length $I_{i}^{(\mathrm{M})}$, the initial mild infective $i$ makes contact with the initial susceptibles at the points of $\eta_{i}^{(\mathrm{M})}$, and with the initial mild infectives at the points of $n m^{-1} \xi_{i}^{(\mathrm{M})}$. (If $\xi$ is a simple point process on $[0, \infty)$ with points at $t_{1}<t_{2}<\cdots$, then the point process with points at $\alpha t_{1}<\alpha t_{2}<\cdots, \alpha>0$, is denoted by $\alpha \xi$, which hence has a rate changed by a factor of $1 / \alpha$.) For $k=1,2, \ldots$, the $k$ th contact made with the initial susceptibles is with individual $\chi_{k}^{(n)}$, and the $k$ th contact made with the initial mild infectives is with individual $\tilde{\chi}_{k}$. For $k=1,2, \ldots$, if the $k$ th contact occurring in $E_{n}$ is with a susceptible then that individual becomes mildly infective if $U_{k}<p_{\mathrm{M}}$ (otherwise the contact is ignored); if it is with a mild infective then that individual becomes a severe case if $U_{k}<p_{\mathrm{S}}$ (otherwise the contact is ignored); and if it is with an immune individual then nothing happens. The $k$ th susceptible infected by the epidemic adopts the set of random quantities $\mathcal{H}_{k}$; if $t$ denotes this individual's time of infection then it makes contacts during $\left[t, t+I_{k}^{(\mathrm{M})}\right]$ at times given by $\left\{t+\eta_{k}^{(\mathrm{M})}\right\} \cup\left\{t+n m^{-1} \xi_{k}^{(\mathrm{M})}\right\}$. Suppose that a mild case (governed by $\mathcal{H}_{k}$, say) becomes a severe case at time $t$; then, in addition to any contacts remaining from this individual's mild infectious period, it makes contacts during $\left[t, t+I_{k}^{(\mathrm{S})}\right]$ at times given by $\left\{t+\eta_{k}^{(\mathrm{S})}\right\} \cup\left\{t+n m^{-1} \xi_{k}^{(\mathrm{S})}\right\}$. The epidemic stops when there are no remaining mild or severe cases in the population.

The above random quantities can also be used to define a realisation of a Crump-Mode-Jagers branching process, with $m$ initial ancestors labelled $-(m-1),-(m-2), \ldots, 0$, as follows. 
For $i=-(m-1),-(m-2), \ldots, 0$, initial ancestor $i$ lives for time $I_{i}^{(\mathrm{M})}$ and has potential births at times given by $\eta_{i}^{(\mathrm{M})}$. For $k=1,2, \ldots$, the $k$ th potential birth in the branching process gives rise to a new individual if $U_{k} \leq p_{\mathrm{M}}$. Suppose that the $k$ th actual birth in the branching process occurs at time $t>0$. Then that individual lives until time $t+I_{k}^{(\mathrm{M})}$ and has potential births at times given by $\left\{t+\eta_{k}^{(\mathrm{M})}\right\} \cap\left[t, t+I_{k}^{(\mathrm{M})}\right]$. For $t \geq 0$, let $Y_{n}(t)$ denote the number of mildly infectious individuals in the epidemic $E_{n}$ at time $t$ and let $Y(t)$ denote the number of individuals alive in the branching process at time $t$. Observe that $Y_{n}(t)$ and $Y(t)$ coincide at least up until the first time a contact is made with an individual that is not susceptible.

For $n=1,2, \ldots$, let

$$
M_{n}=\min \left\{k \geq 2: \chi_{k}^{(n)} \in\left\{\chi_{1}^{(n)}, \chi_{2}^{(n)}, \ldots, \chi_{k-1}^{(n)}\right\}\right\} .
$$

Note that this is the 'birthday problem' and it is well known (see, for example, Aldous (1985, p. 96)) that $n^{-1 / 2} M_{n} \stackrel{\mathrm{D}}{\rightarrow} M$ as $n \rightarrow \infty$, where $M$ is a random variable with probability density function $f(x)=x \exp \left(-\frac{1}{2} x^{2}\right), x>0$. As in the proof of Theorem 2.1 of Ball and Donnelly (1995), the Skorokhod representation theorem implies that we may assume the $\chi_{i}^{(n)}, n=1,2, \ldots, i=1,2, \ldots$, to be constructed such that $n^{-1 / 2} M_{n} \stackrel{\text { a.s. }}{\longrightarrow} M$ as $n \rightarrow \infty$, where $M$ is now also defined on $(\Omega, \mathcal{F}, \mathrm{P})$. Let $A_{\mathrm{E}} \in \mathcal{F}$ denote the set on which the branching process $\{Y(t), t \geq 0\}$ becomes extinct and let $Z$ denote the total progeny of the branching process, excluding the $m$ initial ancestors. (Thus, $Z(\omega)<\infty$ if and only if $\omega \in A_{\mathrm{E}}$.)

Theorem 2. (a) For P-almost all $\omega \in A_{\mathrm{E}}$, as $n \rightarrow \infty$,

(i) $Z_{n}^{(\mathrm{M})} \rightarrow Z$ and

(ii) $Z_{n}^{(k)} \rightarrow 0, k=0, \mathrm{~S}$.

(b) For P-almost all $\omega \in \Omega \backslash A_{\mathrm{E}}, Z_{n}^{(\mathrm{M})} \rightarrow \infty$ as $n \rightarrow \infty$.

Proof. Suppose that $\omega \in A_{\mathrm{E}}$ and let $Z_{p}(\omega)$ denote the total number of potential births that take place in the branching process. Then, for almost all $\omega \in A_{\mathrm{E}}, Z_{p}(\omega)<\infty$ and $M_{n}(\omega)>\frac{1}{2} n^{1 / 2} M(\omega)$ for all sufficiently large $n$. Thus, for such $\omega$ and all sufficiently large $n$, every birth in the branching process corresponds to a new mildly infectious case in the epidemic $E_{n}$. For $i=-(m-1),-(m-2), \ldots$, let $W_{n, i}$ be the time, from its initial infection until it contacts an initial infective, that the individual governed by $\mathcal{H}_{i}$ has to wait in $E_{n}$. Note that $W_{n, i} \stackrel{\text { a.s. }}{\longrightarrow} \infty, i=-(m-1),-(m-2), \ldots$, as $n \rightarrow \infty$. Hence, for P-almost all $\omega \in A_{\mathrm{E}}$, no contacts are made with initial infectives in $E_{n}$ for all sufficiently large $n$, and part (a) of the theorem follows. Part (b) is proved by noting that the above argument implies that, for almost all $\omega \in \Omega \backslash A_{\mathrm{E}}$ and each $\ell \in \mathbb{N}$, if $Z(\omega) \geq \ell$ then $Z_{n}^{(\mathrm{M})}(\omega) \geq \ell$ for all sufficiently large $n$.

Theorem 2 enables the distribution of the size of a minor outbreak to be approximated for large $n$. We now determine the asymptotic distribution of the final outcome of a major outbreak. Let $p_{\mathrm{E}}$ denote the extinction probability of the branching process $\{Y(t), t \geq 0\}$.

Lemma 2. Let $\left(b_{n}\right)$ be any sequence of real numbers such that $b_{n} \rightarrow \infty$ and $n^{-1} b_{n} \rightarrow 0$ as $n \rightarrow \infty$. Then

$$
\lim _{n \rightarrow \infty} \mathrm{P}\left[Z_{n}^{(\mathrm{M})} \leq b_{n}\right]=p_{\mathrm{E}}
$$

Proof. For $k=1,2, \ldots$,

$$
\liminf _{n \rightarrow \infty} \mathrm{P}\left[Z_{n}^{(\mathrm{M})} \leq b_{n}\right] \geq \liminf _{n \rightarrow \infty} \mathrm{P}\left[Z_{n}^{(\mathrm{M})} \leq k\right]=\mathrm{P}[Z \leq k]
$$


by Theorem 2. Letting $k \rightarrow \infty$ in (20) yields

$$
\liminf _{n \rightarrow \infty} \mathrm{P}\left[Z_{n}^{(\mathrm{M})} \leq b_{n}\right] \geq p_{\mathrm{E}}
$$

For $\varepsilon \in(0,1)$, let $\left\{Y_{\varepsilon}(t), t \geq 0\right\}$ be the branching process constructed from $\{Y(t), t \geq 0\}$ by ignoring births in $\{Y(t), t \geq 0\}$ independently and with probability $\varepsilon$. Let $p_{\mathrm{E}}(\varepsilon)$ denote the extinction probability of $\left\{Y_{\varepsilon}(t), t \geq 0\right\}$. Following Whittle (1955), note that if $Z_{n}^{(\mathrm{M})} \leq \varepsilon n$ then the branching process $\left\{Y_{\varepsilon}(t), t \geq 0\right\}$ is a lower bound for $\left\{Y_{n}(t), t \geq 0\right\}$. Thus, if $Z(\varepsilon)$ denotes the total progeny of $\left\{Y_{\varepsilon}(t), t \geq 0\right\}$ (not including the initial ancestors), then

$$
\limsup _{n \rightarrow \infty} \mathrm{P}\left[Z_{n}^{(\mathrm{M})} \leq b_{n}\right] \leq \limsup _{n \rightarrow \infty} \mathrm{P}\left[Z\left(n^{-1} b_{n}\right) \leq b_{n}\right] \leq \limsup _{n \rightarrow \infty} p_{\mathrm{E}}\left(n^{-1} b_{n}\right) .
$$

Now, $p_{\mathrm{E}}\left(n^{-1} b_{n}\right) \rightarrow p_{\mathrm{E}}$ as $n \rightarrow \infty$, so we have

$$
\limsup _{n \rightarrow \infty} \mathrm{P}\left[Z_{n}^{(\mathrm{M})} \leq b_{n}\right] \leq p_{\mathrm{E}}
$$

The lemma follows from (21) and (22).

We now give a more precise definition of a major outbreak. For $n=1,2, \ldots$, the epidemic $E_{n}$ is said to give rise to a major outbreak if it infects at least $\log n$ susceptibles, i.e. if the event $G_{n}=\left\{Z_{n}^{(\mathrm{M})} \geq \log n\right\}$ occurs. It follows from Theorem 2 and its proof that $\mathrm{P}\left[G_{n}\right] \rightarrow 1-p_{\mathrm{E}}$ as $n \rightarrow \infty$.

Let $R_{0}=\lambda_{\mathrm{M}} p_{\mathrm{M}} \iota_{\mathrm{M}}$. It then follows from standard branching process theory that $p_{\mathrm{E}}<1$ if and only if $R_{0}>1$. Suppose that $R_{0}>1$ and let $\tau=\min \{t>0, t=a(t)\}$. Recalling (11), for $t \geq 0$ let

$$
\tilde{\boldsymbol{\Sigma}}(t)=\left[\begin{array}{ccc}
\sigma_{A}^{2}(t) & \sigma_{A \mathrm{M}}(t) & \sigma_{A \mathrm{~S}}(t) \\
\sigma_{A \mathrm{M}}(t) & \sigma_{\mathrm{M}}^{2}(t) & \sigma_{\mathrm{MS}}(t) \\
\sigma_{A \mathrm{~S}}(t) & \sigma_{\mathrm{MS}}(t) & \sigma_{\mathrm{S}}^{2}(t)
\end{array}\right],
$$

where $\sigma_{A}^{2}(t)$ is obtained by letting $\mu \rightarrow 0$ in the expression for $\tilde{\sigma}_{A}^{2}(t)$, and let $\tilde{\boldsymbol{B}}(t)=\left[\tilde{b}_{i j}(t)\right]$ be the $3 \times 3$ matrix with elements

$$
\begin{aligned}
& \tilde{b}_{11}(t)=\left(1-a^{\prime}(t)\right)^{-1} a^{\prime}(t), \\
& \tilde{b}_{21}(t)=\left(1-a^{\prime}(t)\right)^{-1} r_{\mathrm{M}}^{\prime}(t), \\
& \tilde{b}_{31}(t)=\left(1-a^{\prime}(t)\right)^{-1} r_{\mathrm{S}}^{\prime}(t), \\
& \tilde{b}_{i j}(t)=0, \quad j>1 .
\end{aligned}
$$

For $n=1,2, \ldots$ let $\tilde{Z}_{n}=\left(A_{\bullet n}\left(\bar{T}_{\infty}^{(n)}\right), Z_{n}^{(\mathrm{M})}, Z_{n}^{(\mathrm{S})}\right)^{\top}$, and for $t \in[0, \infty]$ let

$$
\boldsymbol{\mu}_{\tilde{Z}}(t)=\left(a(t), r_{\mathrm{M}}(t), r_{\mathrm{S}}(t)\right)^{\top} .
$$

Theorem 3. Suppose that $R_{0}>1$, that $\sigma_{i}^{2}<\infty, i=\mathrm{M}, \mathrm{S}$, and that $a^{\prime}(\tau)<1$. Then

$$
\left[n^{-1 / 2}\left(\tilde{\boldsymbol{Z}}_{n}-n \boldsymbol{\mu}_{\tilde{Z}}(\tau)\right) \mid G_{n}\right] \stackrel{\mathrm{D}}{\rightarrow} \tilde{\boldsymbol{Z}} \quad \text { as } n \rightarrow \infty,
$$

where $\tilde{\boldsymbol{Z}}$ is a three-dimensional zero-mean normal random vector with variance-covariance matrix given by

$$
\tilde{\boldsymbol{\Sigma}}_{Z}=(\boldsymbol{I}+\tilde{\boldsymbol{B}}(\tau)) \tilde{\boldsymbol{\Sigma}}(\tau)(\boldsymbol{I}+\tilde{\boldsymbol{B}}(\tau))
$$


Proof. First note that it follows from Lemma 2 that

$$
\lim _{n \rightarrow \infty} \mathrm{P}\left[Z_{n}^{(\mathrm{M})}>b_{n} \mid G_{n}\right]=1
$$

for any sequence $\left(b_{n}\right)$ such that $b_{n} \rightarrow \infty$ and $n^{-1} b_{n} \rightarrow 0$ as $n \rightarrow \infty$. It then follows (see the proof of Theorem 3.12 of Ball and Neal (2003)) that there exists a $b>0$ such that

$$
\lim _{n \rightarrow \infty} \mathrm{P}\left[Z_{n}^{(\mathrm{M})}>b n \mid G_{n}\right]=1 .
$$

Observe that Lemma 1 holds in the present setting, with $\mu=0$, implying that $\tilde{a}(t)=a(t), t \in$ $[0, \infty]$. The equation $a(t)=t$ has roots $t=0$ and $t=\tau$, and arguing as in the proof of Corollary 1 shows that

$$
\min \left\{\bar{T}_{\infty}^{(n)},\left|\bar{T}_{\infty}^{(n)}-\tau\right|\right\} \stackrel{\text { a.s. }}{\longrightarrow} 0 \quad \text { as } n \rightarrow \infty .
$$

Recall that $Z_{n}^{(\mathrm{M})}=R_{\bullet n}^{(\mathrm{M})}\left(\bar{T}_{\infty}^{(n)}\right)$, so, again arguing as in the proof of Corollary 1, we find that

$$
\left[\bar{T}_{\infty}^{(n)} \mid G_{n}\right] \stackrel{\mathrm{P}}{\rightarrow} \tau \quad \text { and }\left[Z_{n}^{(i)} \mid G_{n}\right] \stackrel{\mathrm{P}}{\rightarrow} r_{i}(\tau), \quad i=\mathrm{M}, \mathrm{S}, \quad \text { as } n \rightarrow \infty
$$

(The zero root of $a(t)=t$ is excluded since $\lim _{n \rightarrow \infty} \mathrm{P}\left[Z_{n}^{(\mathrm{M})}>b n \mid G_{n}\right]=1$.)

A realisation of $\left[\tilde{\boldsymbol{Z}}_{n} \mid G_{n}\right]$ can be constructed as follows. Construct the epidemic $E_{n}$ in real time using the construction described at the start of this subsection. Stop this construction as soon as $\log n$ susceptibles have been infected, and let $T_{n}^{*}$ denote the sum of the remaining mild infectious periods of all those individuals that are infectious at that time. Now use the embedding construction of Section 2.3.1 with $m+[\log n]+1$ initial mild infectives, which exert $T_{0}^{(n)}=\lambda_{\mathrm{M}} T_{n}^{*}$ units of infectious pressure on the population, and $n-\lfloor\log n\rfloor-1$ susceptibles, where $\lfloor\cdot\rfloor$ denotes the integer-part function. Note that $n^{-1 / 2} T_{0}^{(n)} \stackrel{\text { a.s. }}{\longrightarrow} 0$ as $n \rightarrow \infty$. Theorem 3 now follows by an argument similar to that in the proof of Theorem 1 , since $\left[\bar{T}_{\infty}^{(n)} \mid G_{n}\right] \stackrel{\mathrm{P}}{\rightarrow} \tau$ as $n \rightarrow \infty$.

\section{Discussion}

The EDS model of this paper is designed to describe a transmittable disease that might have different severities, with these severities also affecting the amount of possible further transmission. The model does so by letting infected individuals first become mildly infectious and later, if exposed sufficiently further to the disease, become severely infectious. This exposuredependent severity is one way of modelling the phenomenon of interest. A perhaps less realistic feature of the model is that the severe infectious state can occur during and/or after the mild infectious state - as mentioned above, 'additionally infectious' might be a better name for the second state. It would perhaps be more realistic to allow for an increased infectivity during the infectious period if exposed to enough additional exposure, but to have this increase in infectivity stop when the (original) infectious period ends. However, such a model is less tractable from a mathematical point of view.

An alternative model that may capture the same feature is one in which each individual can become only mildly or severely infected (and not both, as in the EDS model), and in which the chances that a contacted individual becomes mildly or severely infected depend on the state of the individual transmitting the disease. A possible advantage of such an infector-dependent severity model is that an infected individual is only ever in one of the two infectious states; 
however, this might also be a disadvantage: once an individual has been infected, the infection severity might very well depend on possible extra exposure. The authors intend to study a model with such an infector-dependent severity in a forthcoming paper.

The present model permits only two types of severity, mild and severe. It is mathematically straightforward to extend the present model to allow for several severity stages. The same qualitative features remain, i.e. the reproduction number, $R_{0}$, depends only on parameters of the mildest state, whereas the final size, in the case of a major outbreak, depends on the parameters of all states, and possibly in a discontinuous way. In fact, the model can be extended further to allow the severity to depend continuously on an individual's exposure to the disease, by defining the model directly in terms of the functions $A_{i}$ of Section 4.1 and noting that (subject to a mild moment condition) the proofs still hold if the sample paths of $A_{i}$ are càdlàg and nondecreasing.

Important problems in preventing infectious disease outbreaks, beside modelling the epidemic, are statistical inference and the study of the effects of vaccination. Neither of these problems has been addressed in the present paper. In particular, an interesting open problem is to model and analyse how vaccination affects susceptibility and potential severity, and how this in turn affects $R_{0}$. The fact that the final size may be discontinuous in the model parameters indicates that interesting features may also be found when analysing the effects of vaccination. Indeed, the simulations described in Section 3.2 suggest that the reduction of $R_{0}$ to below its usual critical value of 1 may not be sufficient to prevent major outbreaks.

\section{Acknowledgements}

This research was supported by the Swedish Research Council. We thank the organisers and participants of the Mariefred Epidemic Workshop in 2003.

\section{References}

Aaby, P., Bukh, J., Lisse, I. M. AND DA Silva, M. C. (1988). Further community studies on the role of overcrowding and intensive exposure on measles mortality. Rev. Infect. Dis. 10, 474-477.

Aldous, D. J. (1985). Exchangeability and related topics. In Ecole d'Été de Probabilités de Saint-Flour XIII (Lecture Notes Math. 1117), ed. P. L. Hennequin, Springer, Berlin, pp. 1-198.

Andersson, H. And Britton, T. (2000). Stochastic Epidemic Models and Their Statistical Analysis (Lecture Notes Statist. 151). Springer, New York.

Ball, F. G. And Donnelly, P. J. (1995). Strong approximations for epidemic models. Stoch. Process. Appl. 55, 1-21.

Ball, F. G. And Neal, P. J. (2003). The great circle epidemic model. Stoch. Process. Appl. 107, 233-268.

ButLer, J. C. et al. (1994). Household-acquisition of measles and illness severity in an urban community in the United States. Epidemiol. Infect. 112, 569-577.

Greenhalgh, D., Diekmann, O. and de Jong, M. C. M. (2000). Subcritical endemic steady states in mathematical models for animal infections with incomplete immunity. Math. Biosci. 165, 1-25.

Heesterbeek, J. A. P. And Dietz, K. (1996). The concept of $R_{0}$ in epidemic theory. Statist. Neerlandica 50, 89-110.

LEFÈvre, C. (1990). Stochastic epidemic models for S-I-R infectious diseases: a brief survey of the recent theory. In Stochastic Processes in Epidemic Theory (Lecture Notes Biomath. 86), eds J.-P. Gabriel, C. Lefèvre and P. Picard, Springer, Berlin, pp. 1-12.

Mangada, M. N. M. AND Igarashi, A. (1998). Molecular and in vitro analysis of eight dengue type 2 viruses isolated from patients exhibiting different disease severities. Virology 244, 458-466.

Medley, G. F., Lindop, N. A., Edmunds, W. J. And NoKes, D. J. (2001). Hepatitis-B virus endemicity: heterogeneity, catastrophic dynamics and control. Nature Medicine 7, 619-624.

Morley, D. C. AND Aaby, P. (1997). Managing measles: size of infecting dose may be important. British Medical J. 314, 1692.

Parang, N. M. And Archana, C. (2004). Varicella. eMedicine September 15, 2004. Available at http://www.emedicine. com/ped/topic2385.htm.

Scalia-Tomba, G.-P. (1985). Asymptotic final size distribution for some chain-binomial models. Adv. Appl. Prob. 17, $477-495$. 
Scalia-Tomba, G.-P. (1990). On the asymptotic final size distribution of epidemics in heterogeneous populations. In Stochastic Processes in Epidemic Theory (Lecture Notes Biomath. 86), eds J.-P. Gabriel, C. Lefèvre and P. Picard, Springer, Berlin, pp. 189-196.

SellKe, T. (1983). On the asymptotic distribution of the size of a stochastic epidemic. J. Appl. Prob. 20, 390-394.

VAN DEN DRIEssche, P. AND WatMough, J. (2002). Reproduction numbers and sub-threshold endemic equilibria for compartmental models of disease transmission. Math. Biosci. 180, 29-48.

VAN Der VAart, A. W. AND Wellner, J. A. (1996). Weak Convergence and Empirical Processes. With Applications to Statistics. Springer, New York.

Whittle, P. (1955). The outcome of a stochastic epidemic - a note on Bailey's paper. Biometrika 42, 116-122. 